\title{
Intention Recognition for Dynamic Role Exchange in Haptic Collaboration
}

\author{
Ayse Kucukyilmaz, and T. Metin Sezgin, and Cagatay Basdogan
}

\begin{abstract}
In human-computer collaboration involving haptics, a key issue that remains to be solved is to establish an intuitive communication between the partners. Even though computers are widely used to aid human operators in teleoperation, guidance, and training, since they lack the adaptability, versatility, and awareness of a human, their ability to improve efficiency and effectiveness in dynamic tasks is limited. We suggest that the communication between a human and a computer can be improved if it involves a decision making process in which the computer is programmed to infer the intentions of the human operator and dynamically adjust the control levels of the interacting parties to facilitate a more intuitive interaction setup. In this paper, we investigate the utility of such a dynamic role exchange mechanism where partners negotiate through the haptic channel to trade their control levels on a collaborative task. We examine the energy consumption, the work done on the manipulated object, and the joint efficiency in addition to the task performance. We show that when compared to an equal control condition, a role exchange mechanism improves task performance and the joint efficiency of the partners. We also show that augmenting the system with additional informative visual and vibrotactile cues, which are used to display the state of interaction, allows the users to become aware of the underlying role exchange mechanism and utilize it in favor of the task. These cues also improve the user's sense of interaction and reinforce his/her belief that the computer aids with the execution of the task.
\end{abstract}

Index Terms-Human-Computer Interaction, Haptic Collaboration, Haptic Guidance, Haptic User Interfaces, Human Factors, Multimodal Systems, Virtual Environment Modeling, Dynamic Role Exchange, Intention Recognition, Performance Metrics.

\section{INTRODUCTION}

$\mathrm{H}$ APTIC collaboration requires partners to actively adapt to changes in one another's requirements and construct a shared knowledge base about the operations and intentions of each other [1]. In this study, we investigate the necessary components that enhance various aspects of haptic interaction in highly dynamic collaborative tasks. Human-robot interaction, computer aided design, simulation-based medical training, and interactive games can be listed as potential applications for schemes that implement different roles for the human and the computer partners. We believe that in a collaborative system, the human and the computer need to partition the task into units to get maximum benefit from each other's abilities. For example, in [2], Subasi and Basdogan illustrate a good example of human-computer collaboration in molecular docking. In their application, the human operator manipulates a small molecule in a virtual environment through a haptic device to search for the true binding cavity on the surface of a large molecule. Once the binding site is discovered, the computer takes over the control and fine-tunes the alignment of the molecule inside the cavity. In general, it is assumed that humans are good at tasks that require perceptual and cognitive processing, and benefit greatly from prior knowledge. On the other

The authors are with the College of Engineering, Koc University, Sariyer, Istanbul, 34450, Turkey.

E-mail: akucukyilmaz@ku.edu.tr, mtsezgin@ku.edu.tr, cbasdogan@ku.edu.tr hand, computers are widely accepted to be superior in tasks that require precision and accuracy. Hence, we believe that a collaborative scheme will yield the best results if it successfully divides the labor of the computer and the human regarding their strong abilities and dynamically let each party to take control in appropriate moments during the task.

Our goal in this study is to enable intuitive haptic communication between humans and computers in collaborative systems. One way of achieving such communication is to change the degree of partners' control levels dynamically during the task, regarding the intentions of the human. We propose a haptic role exchange mechanism to realize control trade between partners. Also, we suggest that the interaction experience can be further enhanced if the state of interaction is explicitly conveyed to the human operator. In order to achieve this, informative cues, in various forms and through multiple modalities, can be used to signal the interaction state. Although displaying arbitrary combinations of such cues may hamper communication if perceptive or cognitive conflicts arise in the process [3], an effective combination of these cues can be beneficial. Even though existing studies demonstrate that the use of multiple modalities improves interaction in virtual environments [4], to our knowledge, no study has been conducted to utilize multimodal feedback to inform the users on different states of the system to facilitate collaborative interaction through the haptic channel.

In this study, we extend the work presented in [5] to 
further evaluate the benefits and the usability of a role exchange mechanism as a shared control scheme. As a test bed, we used a target-hitting task, and developed a model for haptic collaboration in which the human and the computer interact through force to achieve a common goal by dynamically exchanging roles. Additional sensory cues are also integrated to inform the human operator about the current role of each party during the task. The proposed role exchange scheme is compared with an equal control guidance method.

For evaluation, we establish quantitative metrics such as: task completion time, number of errors made during the task, energy consumed by the partners, and work done on the manipulated object. We also suggest a metric to quantify the efficiency. We show that the proposed role exchange mechanism improves task performance and the efficiency of the user as well as the joint efficiency of the partners. Furthermore, the additional sensory cues, which are used to display the control state of the collaborating parties, increase the user's awareness, perceived level of interaction, and reinforce his/her belief that the computer helps with the execution of the task.

In the next section, we summarize the related work in literature. Section 3 presents the haptic negotiation model and discusses its use for dynamic and natural collaborative decision making. The test bed application is introduced in Section 4. The experimental conditions and design is explained along with the measures in Section 5. Section 6 summarizes our results, and finally Section 7 presents conclusions and future directions.

\section{BACKGROUND}

Haptics has been widely used to implement computer guidance in training applications, where the main goal is to teach the trainees the dynamics of the task [6], [7], [8], [9], [10]. Recently, researchers showed that haptic guidance systems can be further improved if they are equipped with predictive and progressive mechanisms [11], [12], [13]. Also cooperative shared control schemes were developed for haptic systems, where humans and computers share the control of a system to collaborate towards a common goal (See [14] for an extensive review). Even though shared control provides a good interface between a human and a computer, its applications in haptics fail to define different autonomy levels for the operators. Adjustable autonomy has been used in multi-agent systems for a decade to make teamwork more effective by interfacing the user with a remote robot at variable autonomy levels [15], [16].

Although the idea of adjustable autonomy has not yet matured in the context of haptic collaboration, the notion of exchanging roles and trading control levels have arisen. Many researchers examined role definitions in haptic human-human interaction. Nudehi et al. [17] developed a system for two collaborating humans. This system switched the "control authority" to one party regarding the difference in the individuals' actions. Reed and Peshkin [18] observed two different specialization behaviors -accelerator and deceleratorwhen two humans interact in a 1-DOF target acquisition task. Feth et al. [19] presented an analysis that associates the energy flow between interacting partners with role distributions. Stefanov et al. [20] defined non-exclusive executor and conductor roles and proposed a model for analyzing the roles during the task. They successfully determined the roles of the parties using the velocities and the interaction forces that are applied through the haptic devices. Groten et al. [6] investigated the effect of haptic interaction in not only collaborative but also conflicting decision situations. In this study, the users were instructed to agree/disagree with the operation of their partner or to remain passive and obey their partner in a path following task. They observed that in the existence of disagreements, task performance deteriorates and the amount of physical effort is increased. This increase was interpreted as the additional negotiation effort.

Despite the studies in human-computer collaboration, there are limited number of studies on role definitions and assignments for human-computer interaction involving haptics. Evrard et al. [21] implemented a role exchange mechanism in a symmetric dyadic task where a human interacts with a computer. They defined leader and follower roles and used two functions to define each operator's control level on the task by setting certain weight parameters. Although this model ensured a systematic and smooth transition between roles, the interaction was not designed to be user-centric, and did not involve dynamic negotiation. Corteville et al. [22] used a velocity based dominance factor to adjust the assistance level in a 1-DOF pointto-point movement task. The assistance level was set based on an estimate of the motion characteristics of the human in a known trajectory. However, the amount of assistance was predetermined and did not change dynamically during the task. In [23], Duchaine and Gosselin implemented a variable impedence control scheme for human-computer collaboration. In order to identify the parameters of this scheme, they utilized the time derivative of human force as an indicator of the human's intention of accelerating or decelerating.

\section{Haptic Negotiation model and the Role Exchange Mechanism}

In this section, we describe our haptic negotiation model, which constitutes the basis of our role exchange mechanism. This model allows the interaction of two agents (i.e. a human and a computer) through a negotiated interface point. The haptic negotiation 
model is sketched in Fig. 1. The interaction is implemented using three massless particles and a springdamper model between them. These particles serve as the interface points, through which the parties communicate with the system. The interface points, labeled as HIP and CIP in the figure, respectively denote the human's Haptic Interface Point and the Computer's Interface Point. The operations of the parties are combined by interconnecting these two points at NIP (Negotiated Interface Point), and by allowing NIP to move the manipulated object.

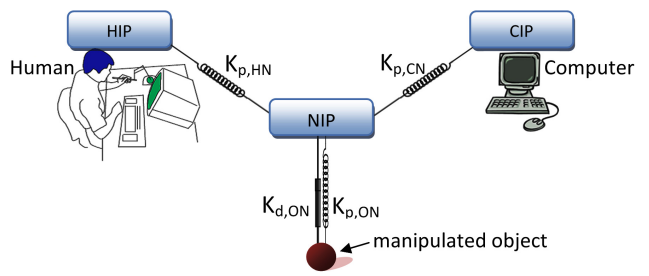

Fig. 1. Our haptic negotiation model. Force negotiation is achieved by setting the stiffness constants, $K_{p, H N}$ and $K_{p, C N}$. These constants are used to adjust the control levels of the parties.

This model allows the control of a virtual point to be shared between parties. It also facilitates the process of assigning different control levels (i.e. roles) to the parties. The human and the computer are granted different levels of control on the task by varying the stiffness coefficients between HIP and NIP $\left(K_{p, H N}\right)$ and between CIP and NIP $\left(K_{p, C N}\right)$. If $K_{p, C N}$ and $K_{p, H N}$ have equal value, the computer and the user will have equal control on the movement of the manipulated object. On the other hand, the computer will be the dominant actor if $K_{p, C N}$ has a larger value, and vice versa, the human will be dominant if $K_{p, H N}$ is larger. $K_{p, O N}$ and $K_{d, O N}$ affect how much and how fast the object is manipulated when NIP is moved under the influence of the forces applied by the human and the computer ${ }^{1}$.

The parties interact through force information to enable the role exchanges. The computer infers the user's intention of taking control and shifts the roles as intended. It is assumed that the user shows his/her intention of taking control by applying large forces, whereas (s)he tries to relinquish control to the computer by reducing the forces (s)he applies. In practice, the user initiates a role exchange whenever the magnitude of the force (s)he applies is above an upper threshold or below a lower threshold over a predetermined period. Note that the forces in our system vary between $0-4 \mathrm{~N}$, hence the term "large force" is relative and indicates that the force applied by the user is higher than the upper force threshold. Upper and lower force thresholds are initially set at the beginning of the game, but new user-specific

1. The stiffness and damping coefficients used in the experiment are as follows: $K_{p, O N}=0.5 \mathrm{~N} / \mathrm{m}, K_{d, O N}=0.0015 \mathrm{Ns} / \mathrm{m}$; $0.05 \mathrm{~N} / m \leq K_{p, H N} \leq 0.45 \mathrm{~N} / \mathrm{m}$ and $K_{p, C N}=0.5 \mathrm{~N} / \mathrm{m}-K_{p, H N}$ during role exchange values are calculated and updated adaptively later during the game:

$$
\begin{aligned}
& \tau_{L}=\mu_{F}-\sigma_{F}, \\
& \tau_{U}=\mu_{F}+\sigma_{F},
\end{aligned}
$$

where $\mu_{F}$ and $\sigma_{F}$ are respectively the average of the forces applied by the user and the standard deviation of these forces.

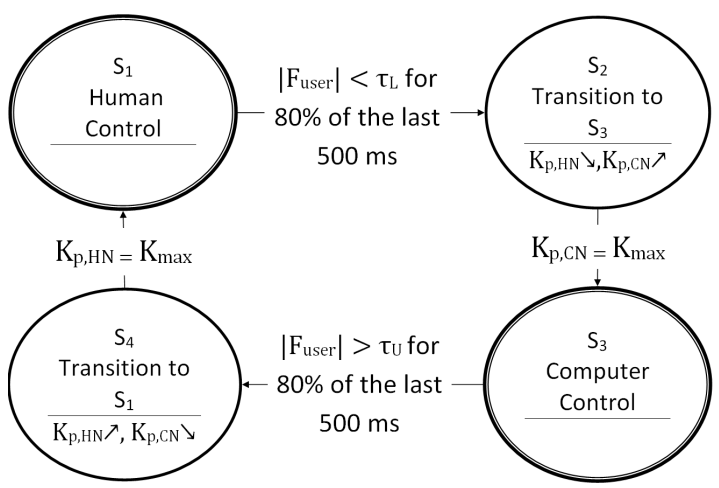

Fig. 2. State diagram defining the role exchange policy. $\left|F_{\text {user }}\right|$ is the magnitude of the force applied by the user.

Fig. 2 illustrates the finite state machine (FSM) used to realize a smooth transition during the role exchanges. The states of the FSM define the interaction states within the system. Human Control $\left(S_{1}\right)$ and Computer Control $\left(S_{3}\right)$ states express the two extreme control levels that are defined in our application. Initially the system is in $S_{1}$, in which the user acts as the controller of the task. If the user wants to, (s)he can give control to the computer. We designed the role exchange mechanism so that the forces applied by the user need to stay below the personalized lower threshold value for more than $80 \%$ of a 500 millisecond duration to initiate a role exchange. Hereby, the user will make the system enter the transition state $S_{2}$, in which the control is gradually shifted to the computer until the predefined control transition period is over (i.e. $750 \mathrm{~ms})^{2}$; only then the system enters the Computer Control state $\left(S_{3}\right)$. Similarly, when the system is in Computer Control state $\left(S_{3}\right)$, the user can decide to take over control by exerting forces larger than the upper threshold. Then, a series of state transitions will occur from $S_{3}$ to the transition state $S_{4}$, and then to the Human Control state $\left(S_{1}\right)$ in succession. In transition states $\left(S_{2}\right.$ and $\left.S_{4}\right)$, the stiffness coefficients are varied linearly over time to let the user and the computer share control in variable degrees.

\section{Application: haptic Board Game}

The haptic board game is designed especially to create a dynamic and interactive environment that mimics

2. The transition interval was selected to be larger than the typical motor response time (reaction time + movement time) of a human operator in reaching tasks $(\tilde{4} 00 \mathrm{~ms})$ to ensure smooth transitions [24]. 
a physical task, in which the human benefits from collaboration with a computer.

\subsection{Rules and Design Considerations}

In the game, the user controls the position of a ball with a PHANToM ${ }^{\circledR}$ Premium haptic device to hit cylinders on a board. The user is asked to move the ball to hit the target cylinder and wait on it to the count of 10. A counter appears in the middle of the screen to alert the user to the countdown status while the ball resides on the target cylinder. If the user succeeds in staying on the target until the end of the countdown, the color of that cylinder changes to red, indicating that it has been collected, and a new target cylinder is determined. This cylinder is colored green to indicate that it is the new target cylinder the user should hit. In Fig. 3, the cylinder in the upper right corner of the board was previously hit, whereas the target cylinder lies at the lower right corner. The remaining two cylinders are unhit. To motivate the users, we display a set of messages on the screen at the end of a trial -after hitting all 4 cylinders on the board. These messages bear either positive (i.e. "Good job", "Much better", "Excellent") or negative (i.e. "You can do better") meaning and are invoked regarding the improvement or deterioration in the user's performance.

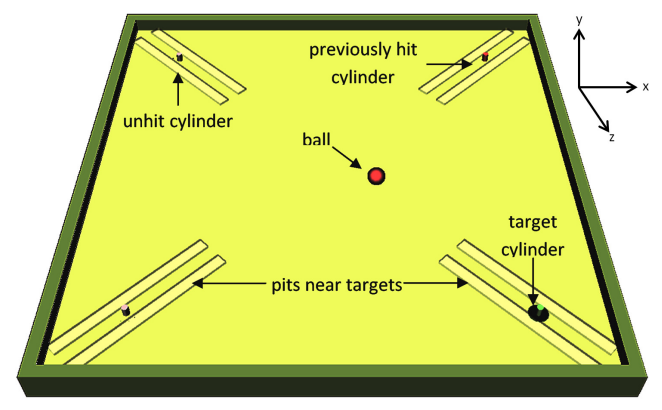

Fig. 3. A screenshot of the haptic board game.

Each cylinder is located in between two pits which diagonally extend towards the center of the board. The users are instructed to avoid falling into these pits. If the ball falls in a pit, that pit is highlighted to warn the user and the ball is imprisoned in the pit. To leave the pit, the user should move the ball towards the entrance of the pit. As an additional penalty, if the ball falls in a pit, all acquired targets are undone, hence the trial is restarted. The pits are designed to serve as "difficult" regions for the human, where the user is anticipated to ask for computer guidance. It is relatively "easy" to control the movements of the ball outside the pits. This design is chosen to create a task where a human and a computer can perform better than one another at different times during the execution of the task. Additionally, as a result of the movement of the ball, the board is tilted about $x$ and $\mathrm{z}$ axes and the users are fed back with forces due to the rotation of the board. This feature makes the game more difficult, hence the users are further motivated to demand computer guidance.

\subsection{Physics-Based Engine}

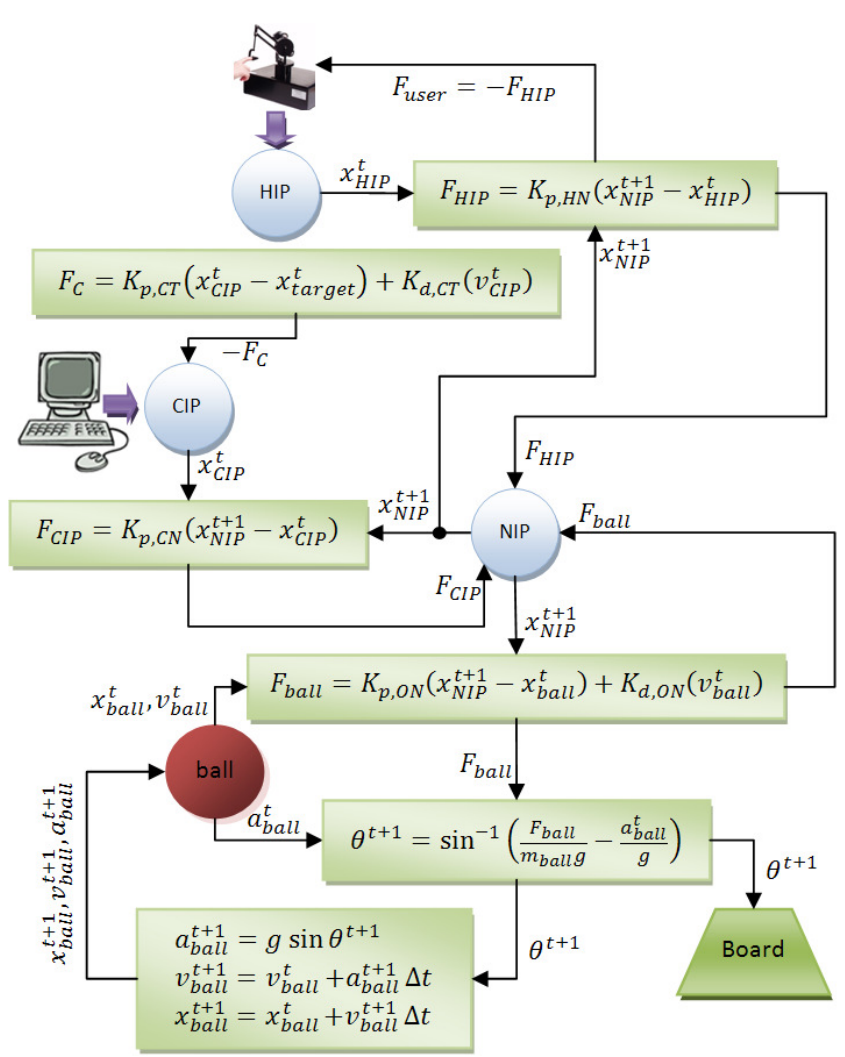

Fig. 4. The flow of interactions within the game.

The physics-based model used for simulating the game dynamics is shown in Fig. 4. At each time step, the controller moves CIP by applying a force $F_{C}$ to reach a target point. This force is calculated using a PD (Proportional-Derivative) control algorithm, for which the proportional and derivative gains $K_{p, C T}$ and $K_{d, C T}$ are set to $5.0 \times 10^{-6} \mathrm{~N} / \mathrm{m}$ and $3.5 \times 10^{-3} \mathrm{Ns} / \mathrm{m}$, respectively. Using the PD controller, CIP is forced to follow a trajectory defined by the via points that are connected to each other by line segments. Three via points are defined for each target as illustrated in Fig. 5. The first via point is used to move CIP to the entrance of the pit. Upon reaching the first via point, the ball is guided by CIP to the target cylinder between the pits with the help of the second via point. Finally, for exiting the pit, CIP leads the ball out of the pit through the use of the third via point.

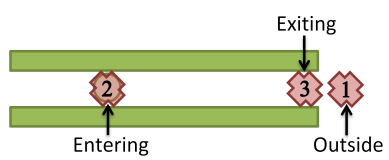

Fig. 5. Three via points are used to define the trajectory of the ball under computer control: at the entrance of the pit (1), on the target (2), and at the exit of the pit (3). 
In our model, the movements of HIP and CIP affect the movements of NIP. At each time step, the new position of NIP $\left(x_{N I P}^{t+1}\right)$ is calculated so that the system illustrated in Fig. 1 is in static equilibrium. The new position of NIP is then used to calculate $F_{C I P}, F_{H I P}$, and $F_{\text {ball }}$. These respectively denote the forces that act on NIP by CIP, HIP, and the ball. $F_{H I P}$ is negated and fed back to the user through the haptic device. The tilt angle of the board $\left(\theta^{t+1}\right)$ is calculated using the force acting on the ball $\left(F_{b a l l}\right)$ and its acceleration $\left(a_{b a l l}^{t}\right)$. Finally, the ball's new position $\left(x_{\text {ball }}^{t+1}\right)$, velocity $\left(v_{\text {ball }}^{t+1}\right)$, and acceleration $\left(a_{\text {ball }}^{t+1}\right)$ are calculated and updated using Euler integration.

\subsection{Additional Sensory Elements}

An advantage of our collaborative model is that it allows the integration of different sensory cues to display the control state. In [25], we observed that when the users are not informed on the nature of the task, some of them fail to understand that their control level within the task is changing. Hence, for this study, we aimed to make the underlying mechanism as visible as possible through the integration of visual and vibrotactile informative cues.

\subsubsection{Visual Cues}

Our application requires users to attend to visual information in order to successfully complete the game. We used two role indication icons, which are displayed over the board to display the control levels of the parties (See Fig. 6). For instance, in Fig. 6(a), the icon for the human (on the right) has greater size, indicating the dominance of the user. Similarly, in Fig. 6(b), the computer's icon (on the left) is larger, indicating that the computer has taken control of the game. The sizes of the icons serve as metaphors for the parties' control levels. The icons are enlarged and shrunk gradually based on the transition process discussed in Section 3 to demonstrate the smooth transition between control levels (i.e. roles). We initially considered locating two role indication bars at each side of the board or a single one on top, which would illustrate the parties' control levels. However, these were not successful in attracting the users' attention. Hence, we selected the icons in Fig. 6 for role-indication.

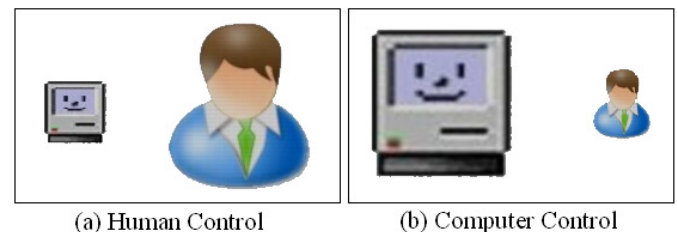

Fig. 6. Two configurations for the role-indication icons.

\subsubsection{Vibrotactile Cues}

In our application, the users rely on force feedback to choose their control actions over the ball movements. Moreover, all negotiation is done through force information. In order to signal the active state of interaction, we used the same channel to display vibrotactile cues to the users. These cues are implemented as vibrations in the y-direction, hence don't interfere with the movement of the ball in any way. Two different types of cues are implemented:

Buzzing is a high frequency vibration $(100 \mathrm{~Hz})$ presented to the user through the haptic device during state $S_{2}$. It signals the initiation and occurrence of role exchange.

Tremor is defined as an involuntary shaking of some body part (i.e. trembling). In order to signal the presence of computer control in state $S_{3}$, a low frequency vibration varying between 8 and $12 \mathrm{~Hz}$ is artificially generated and continuously displayed to the user while the system is in this state.

The choice of the displayed cues and the way they are displayed are not arbitrary. In small-scale pilot studies, we investigated the effectiveness of different cues displayed by different sensory modalities (i.e. vision, sound, and haptics) to convey the control state to the user. However, only the visual and vibrotactile cues in their current forms were found to be effective. In the end, we have chosen to present visual and vibrotactile cues simultaneously to make information processing easier through the acquisition of the same information through multiple channels [26].

\section{EXPERIMENT}

This section presents the experimental conditions, design, and the procedure as well as the measures used in the analyses.

\subsection{Conditions}

Equal Control (EC): The user and the computer share control equally at all times to move the ball. This is achieved by choosing $K_{p, H N}$ and $K_{p, C N}$ constant and equal to each other $\left(K_{p, H N}=K_{p, C N}=0.25 \mathrm{~N} / \mathrm{m}\right)$. In this condition, the user feels guidance forces applied by the controller as well as forces generated due to the dynamics of the game.

Role Exchange (RE): At any point during the game, the user can hand/take over the control of the ball to/from the computer by altering the forces (s)he applies through the haptic device. The computer infers the user's intention of taking over or giving up the control of the game based on the user's force profile and updates its degree of control on the ball.

VisuoHaptic Cues (VHC): As in RE condition, the user can initiate role exchanges to get the computer to dynamically change its degree of control on the ball. Additionally, role-indication icons, buzzing, and tremor are displayed to inform the user about the state of the system. 


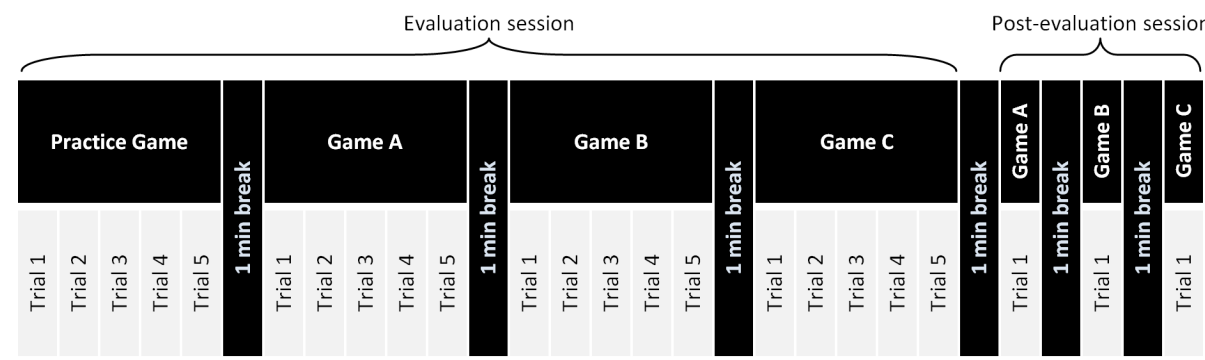

Fig. 7. The order of the conditions displayed to the subjects in the experiment (Practice Game: without assistance; Game A/B/C: one of $\mathrm{EC}, \mathrm{RE}$ or VHC in permuted order)

\subsection{Procedure and Participants}

30 subjects (9 female and 21 male), aged between 21 and 28, participated in our study. All of the subjects were right handed, and they interacted with the haptic device using a thimble attachment. We conducted a within subjects experiment, in which each subject experimented all three conditions in a single day. A practice condition, under which the user plays the game without computer assistance, was initially presented to each subject to familiarize him/her with the system. The guidance conditions (EC, RE, and VHC) were then presented to the subjects in permuted order to balance learning effects. 5 subjects were tested in each of the six permutations of all three guidance conditions. The subjects were given detailed instructions about the conditions. However, since the conditions were presented in mixed order, in order to avoid any perceptual biases, the guidance conditions were labeled as "Game A", "Game B", and "Game $\mathrm{C}^{\prime \prime}$, whereas the practice condition was labeled as the "Practice Game".

The experiment consisted of an evaluation and a post-evaluation session as detailed in Fig. 7. During the evaluation session, the subjects played the haptic board game 5 times (i.e. 5 trials) under each condition. The subjects first played the game without computer guidance, afterwards they played the game under one of the guidance conditions in mixed order. The trial number was displayed in the upper right corner of the game screen. When a trial was over, another trial was started automatically without any interruption until all 5 trials were completed. In each trial, the order of the target cylinders was modified in a controlled manner so that the subjects do not memorize a specific motion path. After playing the game successfully under the same guidance condition for 5 times, a 1minute break was given to the subjects and a new game was started in a different guidance condition. Once the subjects finished the evaluation session, they were given another break before starting the post-evaluation session. In this session, the subjects played the game only once (i.e. one trial only) under each guidance condition in succession (Games A, B, and $C$ ) to remember the conditions and compare the differences in their experiences.

\subsection{Measures}

\subsubsection{Quantitative Measures}

For quantitative analysis, we utilized the data collected during the evaluation session.

We quantify task performance in terms of task completion time and the number of faults made by the user (i.e. how many times a user falls into a pit) in each trial. We also examine the energy consumed by the partners as an indication of physical effort, and the work done to move the ball in order to complete the task (each are normalized with respect to task completion time). Using these two measures, we also introduce an efficiency measure.

Task performance: The completion time of each trial and the total number of faults are counted as performance measures.

Consumed energy $(E)$ : Assuming that no energy is stored in the springs at the beginning, the energy consumed by the human partner is calculated by the dot product of the displacement of HIP and the force exerted by the spring located between NIP and HIP as:

$$
E_{H}=\int_{P_{H}}\left|F_{H I P} \cdot d x_{H I P}\right|,
$$

where $P_{H}$ is the path traversed by HIP during the trial. Similarly, the energy consumed by the computer is computed as:

$$
E_{C}=\int_{P_{C}}\left|F_{C I P} \cdot d x_{C I P}\right|,
$$

where $P_{C}$ is the path traversed by CIP during the trial.

Work done on the ball $(W)$ : The work done on the ball is computed regarding the displacement of the ball and the force acting on the ball by the human (or the computer):

$$
\begin{aligned}
& W_{H}=\int_{P_{B}}\left|F_{H I P} \cdot d x_{b a l l}\right|, \\
& W_{C}=\int_{P_{B}}\left|F_{C I P} \cdot d x_{b a l l}\right|,
\end{aligned}
$$

where $P_{B}$ is the path traversed by the ball during the trial. The total work done on the ball by the partners is computed as:

$$
W_{\text {Total }}=\int_{P_{B}}\left|\left(F_{H I P}+F_{C I P}\right) \cdot d x_{\text {ball }}\right| .
$$


Efficiency $(\eta)$ : Efficiency, in its broad sense defines the ability to produce the desired output with minimum expenditure of time or effort. In [27], Groten et al. proposed an efficiency measure for human computer interaction, which related energy and task performance. On the other hand, our efficiency metric takes the performed work and the energy consumption into account; hence it establishes a well defined way of measuring the mechanical efficiency in a physical task. We define efficiency as the work done on the ball divided by the consumed energy:

$$
\eta=\frac{\text { work done on the ball }}{\text { consumed energy }} \text {. }
$$

It should be noted that the individual efficiencies of the partners are independent of the stiffness and the damping coefficients used for the haptic negotiation model, hence it enables us to compare different guidance conditions. Upon closer inspection, we see that the human partner can maximize his/her individual efficiency, $\eta_{H}=\frac{W_{H}}{E_{H}}$, if (s)he does a large amount of work on the ball with a small effort. However, the joint efficiency of the dyad, $\eta_{\text {Total }}=\frac{W_{\text {Total }}}{E_{\text {Total }}}$, is mostly affected by the harmony of the collaborating partners. If, for example, the human partner continuously acts against the will of the computer, both parties will spend significant effort, yet will fail to move the ball. In such a case, even though the effort is high, the work done on the ball will be small; hence the joint efficiency will be low for the dyad.

\subsubsection{Subjective Measures}

After experimenting with each condition, the subjects are given a questionnaire, which is designed with the technique Basdogan et al. [28] have used in the past for investigating haptic collaboration in shared virtual environments. The questionnaire asks users to comment on their experiences under the 3 guidance conditions (EC, RE, and VHC). Some questions are rephrased and asked again within the questionnaire in random order. For the answers, a 7-point Likert scale is used. The questions are asked in the following categories and the average of the subjects' responses to the questions in each category is used for evaluation:

- Performance: 3 questions are asked to the subjects to assess their self-performance.

- Collaboration: 2 questions investigate whether the subjects had a sense of collaborating with the computer or not.

- Role exchange frequency: A single question is asked to evaluate how frequently the subjects performed role exchanges.

- Degree of control: 2 questions ask the subjects about their perceived degree of control on the ball.

- Interaction: 5 questions explore the level of interaction the subjects experienced during the task.
- Haptic cues: 1 question investigates whether haptic cues increased the subjects' awareness of their control level on the ball.

- Visual cues: 1 question investigates whether visual cues increased the subjects' awareness of their control level on the ball.

- Trust: 2 questions investigate if the subjects trusted their computer partner on controlling the ball.

- Ease of use: 2 questions explore if the interface of the system was easy for the subjects to use.

- Role exchange visibility: A single question explores whether or not the subjects could observe the role exchanges during the task.

- Human-likeness: 2 questions ask the subjects whether the forces felt through the device resembled that of a human.

\section{Results and Discussion}

We present the results of the experiments in terms of the quantitative and subjective measures defined in Section 5.3. We also present the role exchange patterns observed under RE and VHC during the experiment.

\subsection{Quantitative Analysis}

During the experiments, we noticed that some of the subjects failed to perform the task as instructed. For instance, in some trials, the subjects worked against the computer and tried to hit the cylinders in the wrong order, causing an increase in their energy consumption; in others, we observed a high number of faults, which causes the game to restart and eventually results in significantly long completion times. Hence, we concluded that the data contains some outliers which should be addressed. Hence, prior to analysis, we detected the outliers in the data for each of the 7 independent quantitative measurements: completion time, number of faults, energy consumed by the human and the computer, the work done on the ball by the human, the computer, and the dyad. The detection of outliers in data is done by examining the boxplots generated by SPSS. In our outlier elimination procedure, we considered the samples as outliers if they were more than 3 interquartile ranges (IQR) away from the lower or upper quartiles. As a result, $1.6 \%$ of the data are identified as outliers and replaced with the grand mean values.

One-way repeated measures ANOVA is used to discover statistically significant effects of the guidance conditions. Mauchly's test was conducted to check if the assumption of sphericity was violated. If so, the degrees of freedom were corrected using HuynhFeldt estimates of sphericity. Finally, post-hoc t-tests with Bonferroni correction were used for the multiple comparisons between conditions to assess which condition pairs exhibit statistically significant differences between one another. 


\subsubsection{Task Performance}
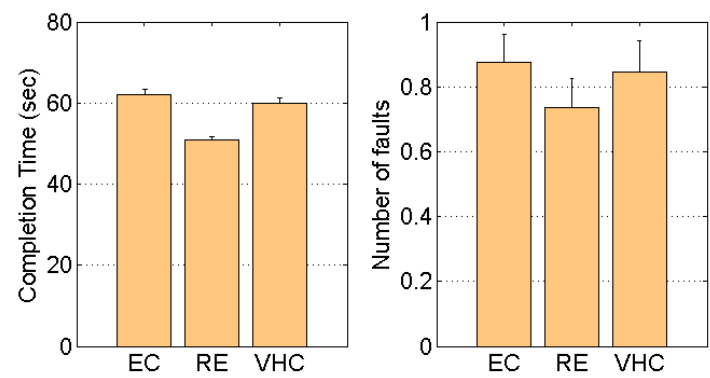

Fig. 8. Average completion time and number of faults under each condition with SEM error bars

Fig. 8 illustrates the mean completion times and the number of faults in each condition and the standard errors of the means (SEM). We observe that the completion time is the shortest under RE, followed by VHC and EC; and the number of faults is the least under RE, again followed by VHC and EC. We observe a significant effect of using the role exchange mechanism on completion time, however a similar effect is not observable on the number of faults done through the task (See Table 1). Although RE condition significantly improves the time performance, adding visual and vibrotactile cues on top of RE, as in VHC, deteriorates the performance significantly probably due to some extra cognitive effort (See Table 2).

\section{TABLE 1}

ANOVA results for the completion time and the number of faults

\begin{tabular}{|l|r|r|r|r|}
\hline Source & $\mathrm{df}$ & $\mathrm{F}$ & $\mathrm{p}$ & $\eta_{\text {partial }}^{2}$ \\
\hline Time & 1.685 & 24.617 & .000 & .142 \\
\hline Faults & 2 & .667 & .514 & .004 \\
\hline
\end{tabular}

TABLE 2

The pairwise comparison of the guidance conditions for the completion time and the number of faults

\begin{tabular}{|l|c|c|c|}
\hline \multirow{2}{*}{} & \multicolumn{3}{|c|}{$\mathrm{p}$-values } \\
\cline { 2 - 4 } & EC-RE & EC-VHC & RE-VHC \\
\hline Time & $.000\left(^{*}\right)$ & .809 & $.000\left(^{*}\right)$ \\
\hline Faults & .759 & 1.000 & 1.000 \\
\hline${ }^{*}$ The mean difference is significant at $\mathrm{p}=.05$ level. \\
\hline
\end{tabular}

\subsubsection{Consumed Energy}

Fig. 9(a) shows the mean values of the energy consumed by the partners and the total energy consumed by the dyad under each condition. The error bars represent the standard error of the means.

We observe a significant effect of the guidance condition on the energy consumed by the human and the computer (See Table 3). We notice that the humans consume significantly more energy under RE and VHC than they do under EC (See Table 4). This indicates that the subjects consume some extra energy when they are presented with a role exchange scheme. Similarly, RE and VHC conditions exhibit similarities in the computer's energy consumption. Finally, we observe that the total energies consumed by the dyad under RE and VHC are significantly more than that of EC. This indicates that, the partners jointly spend more energy under a role exchange scheme.
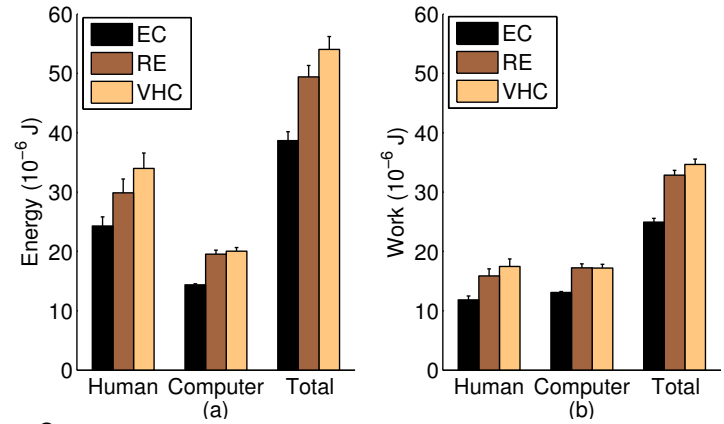

Fig. 9. (a) The average energy consumed and (b) the work done by the human, computer, and the dyad under each condition with SEM error bars

TABLE 3

ANOVA results for the consumed energy $\left(E_{H}\right.$ : energy consumed by human, $E_{C}$ : energy consumed by computer, $E_{\text {Total }}$ : total energy)

\begin{tabular}{|l|r|r|r|r|}
\hline Source & $\mathrm{df}$ & $\mathrm{F}$ & $\mathrm{p}$ & $\eta_{\text {partial }}^{2}$ \\
\hline$E_{H}$ & 2 & 7.950 & .000 & .051 \\
\hline$E_{C}$ & 2 & 48.742 & .000 & .246 \\
\hline$E_{\text {Total }}$ & 1.945 & 27.094 & .000 & .154 \\
\hline
\end{tabular}

TABLE 4

The pairwise comparison of the guidance conditions for the consumed energy

\begin{tabular}{|l|c|c|c|}
\hline \multirow{2}{*}{} & \multicolumn{3}{|c|}{ p-values } \\
\cline { 2 - 4 } & EC-RE & EC-VHC & RE-VHC \\
\hline$E_{H}$ & $.044\left(^{*}\right)$ & $.001\left(^{*}\right)$ & .269 \\
\hline$E_{C}$ & $.000\left(^{*}\right)$ & $.000\left(^{*}\right)$ & 1.000 \\
\hline$E_{\text {Total }}$ & $.000\left(^{*}\right)$ & $.000\left(^{*}\right)$ & .092 \\
\hline
\end{tabular}

\subsubsection{Work Done on the Ball}

In each trial, we computed the work done by the human and the computer on the ball. Fig. 9(b) illustrates the means and the standard errors of the means for the work done on the ball under each condition. The guidance method has a significant effect on the work done by the human, the computer, and the dyad (See Table 5). We note that the work done by the human under VHC and RE are significantly higher than that of EC. Similarly, the amounts of work done by the computer under RE and VHC are significantly higher than that of EC (See Table 6). This is another sign of the similarity in role exchange patterns in RE and VHC. The total work is significantly higher in $\mathrm{VHC}$, followed by RE, and then EC; which means that beside spending more energy, the dyads also colectively do more work under RE and VHC.

\section{TABLE 5}

ANOVA results for the work done on the ball $\left(W_{H}\right.$ : work done by human, $W_{C}$ : work done by computer, $W_{\text {Total }}$ : total work done by the dyad)

\begin{tabular}{|l|r|r|r|r|}
\hline Source & df & $\mathrm{F}$ & $\mathrm{p}$ & $\eta_{\text {partial }}^{2}$ \\
\hline$W_{H}$ & 2 & 15.192 & .000 & .093 \\
\hline$W_{C}$ & 2 & 27.239 & .000 & .155 \\
\hline$W_{\text {Total }}$ & 2 & 84.953 & .000 & .363 \\
\hline
\end{tabular}

In order to understand the role exchange patterns under RE and VHC, we examined the role exchange moments during the task under these two conditions. 


\section{TABLE 6}

The pairwise comparison of the guidance conditions for the work done on the ball

\begin{tabular}{|l|c|c|c|}
\hline \multirow{2}{*}{} & \multicolumn{3}{|c|}{$\mathrm{p}$-values } \\
\cline { 2 - 4 } & EC-RE & EC-VHC & RE-VHC \\
\hline$W_{H}$ & $.042\left(^{*}\right)$ & $.000\left(^{*}\right)$ & .318 \\
\hline$W_{C}$ & $.000\left(^{*}\right)$ & $.000\left(^{*}\right)$ & 1.000 \\
\hline$W_{\text {Total }}$ & $.000\left(^{*}\right)$ & $.000\left(^{*}\right)$ & $.048\left(^{*}\right)$ \\
\hline${ }^{*}$ The mean difference is significant at $\mathrm{p}=.05$ level. \\
\hline
\end{tabular}

Fig. 10 illustrates the positions on the board that the

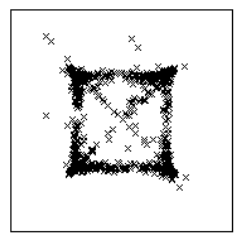

Outside pits

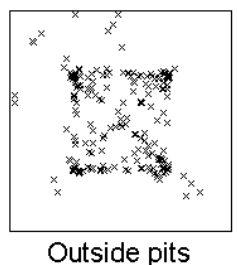

Outside pits

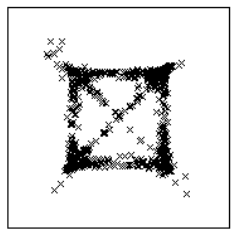

Outside pits

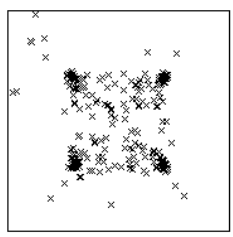

Outside pits

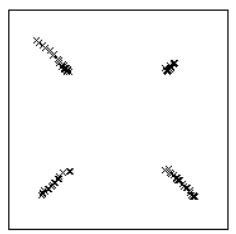

Entering pits

(a) user gets control (RE)

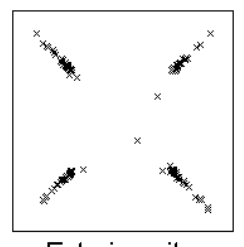

Entering pits

(b) computer gets control (RE)

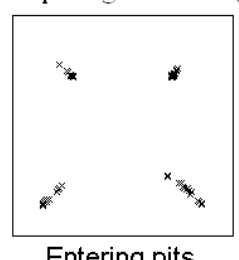

(c) user gets control ( $\mathrm{VHC}$

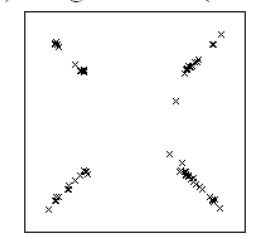

Entering pits

(d) computer gets control (VHC)

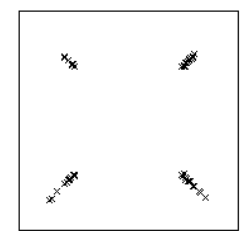

Exiting pits

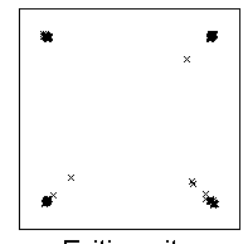

Exiting pits

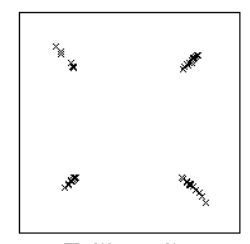

Exiting pits

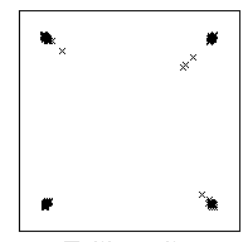

Exiting pits
Fig. 10. The positions on the board where role exchanges were initiated by the user during the game for guidance conditions RE and VHC. Note that the users tend to take control more often outside pit regions, whereas the computer takes control often when entering and exiting pits.

users took control from the computer - 10(a) and 10(c)or handed over control to it - 10(b) and 10(d) - during the experiment. These plots show the distribution of the role exchanges outside pit regions, as well as the distribution at the entrances and exits of the pits. It can be observed from the plots that the pits forced the users to exchange roles: In general, the users took control of the ball outside the pits to move faster. On the other hand, they frequently gave control to the computer when entering and exiting the pits to reduce the number of faults.

Also, we observed that the subjects typically decreased their contribution on the movement of the

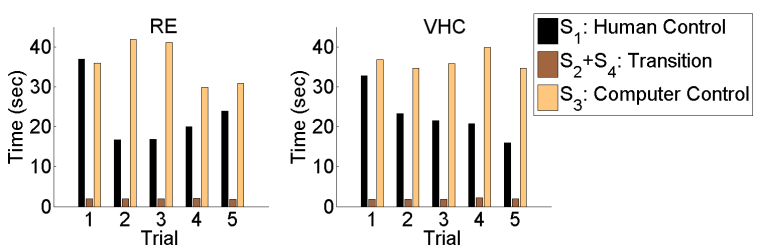

Fig. 11. Average time spent in each state for 5 trials.

ball after the $1^{\text {st }}$ trial, taking advantage of computer guidance until the end of the $5^{\text {th }}$ trial (See Fig. 11). These observations indicate that the computer holds control for similar durations and at similar instants during the task in both RE and VHC.

\subsubsection{Efficiency}

Fig. 12 displays the mean values of the efficiencies and the standard error of the means for each condition. The efficiency of the human under EC is significantly lower than that of both RE and VHC (See Table 8). On the other hand, we observe that the computer's efficiency is maximized under EC. This might suggest that as the subjects take less initiative in performing the task and mostly surrender to computer guidance under EC, the energy consumed by the subjects and their work done is low. As a result of this, the human's efficiency decreases whereas that of the computer increases. However, the joint efficiency of the dyad under RE is significantly higher than that of EC. Even though the difference between the joint efficiencies under RE and VHC is not significant, the joint efficiency of the partners under VHC is slightly lower than it is under RE because of the extra energy consumed by the subjects under this condition.

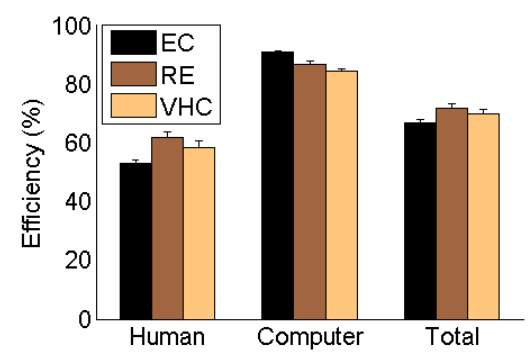

Fig. 12. Average total efficiency and individual efficiencies of the human and the computer under each condition with SEM error bars

TABLE 7

ANOVA results for efficiency ( $\eta_{H}$ : efficiency of human, $\eta_{C}$ : efficiency of computer, $\eta_{\text {Total }}$ : joint efficiency of the dyad)

\begin{tabular}{|l|r|r|r|r|}
\hline Source & $\mathrm{df}$ & $\mathrm{F}$ & $\mathrm{p}$ & $\eta_{\text {partial }}^{2}$ \\
\hline$\eta_{H}$ & 2 & 10.080 & .000 & .063 \\
\hline$\eta_{C}$ & 2 & 19.733 & .000 & .117 \\
\hline$\eta_{\text {Total }}$ & 2 & 3.094 & .047 & .020 \\
\hline
\end{tabular}

\subsection{Subjective Evaluation}

Fig. 13 plots the mean values of the subjects' responses to the questions in the questionnaire, as grouped in Section 5.3.2. Table 9 and 10 present the ANOVA results and $\mathrm{p}$-values for multiple comparisons. 
TABLE 8

The pairwise comparison of the guidance conditions for the efficiency

\begin{tabular}{|l|c|c|c|}
\hline \multirow{2}{*}{} & \multicolumn{3}{|c|}{$\mathrm{p}$-values } \\
\cline { 2 - 4 } & EC-RE & EC-VHC & RE-VHC \\
\hline$\eta_{H}$ & $.000\left(^{*}\right)$ & $.011\left(^{*}\right)$ & .465 \\
\hline$\eta_{C}$ & $.001\left(^{*}\right)$ & $.000\left(^{*}\right)$ & .085 \\
\hline$\eta_{\text {Total }}$ & $.027\left(^{*}\right)$ & .866 & .547 \\
\hline \multicolumn{2}{|c|}{ The mean difference is significant at $\mathrm{p}=.05$ level. } \\
\hline
\end{tabular}

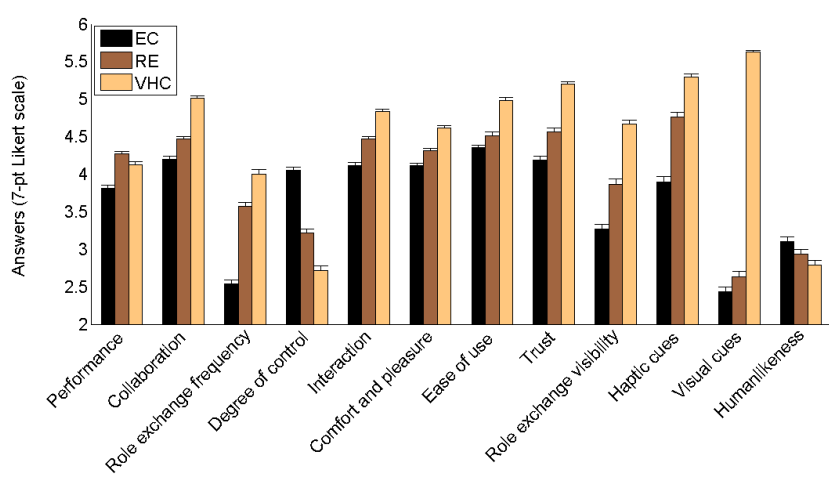

Fig. 13. Means and standard errors of the subjective measures under each guidance condition.

The results can be summarized as follows:

- Performance: The users thought that they achieved the best performance under RE, followed by VHC and EC, however the differences between the conditions are not significant.

- Collaboration: The users reported that the sense of collaboration during the task was the most

TABLE 9

ANOVA results for the subjective measures

\begin{tabular}{|l|r|r|r|r|}
\hline Source & $\mathrm{df}$ & $\mathrm{F}$ & $\mathrm{p}$ & $\eta_{\text {partial }}^{2}$ \\
\hline Performance & 2 & 1.889 & .160 & .061 \\
\hline Collaboration & 2 & 3.847 & .027 & .117 \\
\hline Role ex. frequency & 1.747 & 8.671 & .001 & .230 \\
\hline Degree of control & 2 & 7.992 & .001 & .216 \\
\hline Interaction & 1.736 & 5.315 & .011 & .155 \\
\hline Ease of use & 2 & 3.959 & .024 & .120 \\
\hline Trust & 2 & 4.306 & .018 & .129 \\
\hline Role ex. visibility & 2 & 6.324 & .003 & .179 \\
\hline Haptic cues & 2 & 9.370 & .000 & .244 \\
\hline Visual cues & 1.270 & 53.831 & .000 & .650 \\
\hline Human-likeness & 1.480 & .470 & .571 & .016 \\
\hline
\end{tabular}

TABLE 10

The pairwise comparison of the guidance conditions for the subjective measures

\begin{tabular}{|l|c|c|c|}
\hline \multirow{2}{*}{} & \multicolumn{3}{|c|}{$\mathrm{p}$-values } \\
\cline { 2 - 4 } & EC-RE & EC-VHC & RE-VHC \\
\hline Performance & .208 & .796 & 1.000 \\
\hline Collaboration & 1.000 & .062 & .094 \\
\hline Role ex. frequency & $.031\left(^{*}\right)$ & $.005\left(^{*}\right)$ & .374 \\
\hline Degree of control & .053 & $.005\left(^{*}\right)$ & .265 \\
\hline Interaction & .499 & $.017\left(^{*}\right)$ & .095 \\
\hline Ease of use & 1.000 & $.032\left(^{*}\right)$ & .232 \\
\hline Trust & .963 & $.033\left(^{*}\right)$ & .109 \\
\hline Role ex. visibility & .312 & $.011\left(^{*}\right)$ & .135 \\
\hline Haptic cues & .056 & $.001\left(^{*}\right)$ & .242 \\
\hline Visual cues & .680 & $.000\left(^{*}\right)$ & $.000\left(^{*}\right)$ \\
\hline Human-likeness & 1.000 & 1.000 & 1.000 \\
\hline \multicolumn{4}{|l|}{ * The mean difference is significant at $\mathrm{p}=.05$ level. } \\
\hline
\end{tabular}

under VHC, followed by RE and EC, however the differences between the conditions are not significant.

- Role exchange frequency: The subjects believed that they effectively utilized the role exchange mechanism when applicable and they performed role exchanges equally frequently under RE and VHC.

- Degree of control: Under both RE and VHC, on the average, the subjects held control of the ball for about $37 \%$ of the total duration of the task. However, their perceived degree of control on the ball under EC is significantly higher than that of only VHC. This indicates that even though their control levels were similar under RE and VHC, the subjects could not clearly distinguish between different control levels when no informative cues were present. Hence, we conclude that the additional cues are successful in increasing the user's awareness.

- Interaction: The results suggest that the level of interaction during the task is significantly higher when additional sensory cues are displayed to the users to signal the control state (VHC).

- Ease of use: The results suggest that the interface is significantly easier to use when additional cues are present (VHC).

- Trust: Role exchange let the users trust in their computer partner during collaboration, such that they believe that the computer would move the ball correctly when needed. This sense of trust is significantly higher when additional sensory cues are present (VHC).

- Role exchange visibility, the effect of additional sensory cues: The subjects reported that when additional cues were present, the role exchange process was significantly more visible and they could understand the current state of the system better. They also reported that both visual and vibrotactile haptic cues were effective in enabling them to understand which party had control on the task. However, the visual cues are dominantly preferred by the subjects to determine the control state over the vibrotactile haptic cues.

- Human-likeness: The users reported that none of the guidance conditions created a sense of interacting with a human partner. Interestingly, more than half of the subjects verbally stated that the control provided by the computer was too smooth to be human-like.

\section{Conclusions}

This paper summarizes the results of an experimental study on the utility of a role exchange mechanism as a dynamic and personalized framework for humancomputer collaboration. In this framework, a human dynamically interacts with a computer partner by communicating through the haptic channel to trade control levels on the task. 
In our approach, we used user-specific and dynamically adaptable force thresholds to initiate role exchanges. As the adaptation was done transparently, and since the range for the thresholds was narrow due to the limited output capacity of the haptic device, no user reported any inconsistency or difficulty in adapting to the newly calculated thresholds during the experiment. In this study, we assumed that the computers are better than humans in terms of precision, hence it is reasonable to give control to the computer in case the user decreases the forces (s)he applies as an attempt to do fine-positioning. In order to take over the control, the users were required to generate only a sufficiently large displacement to exceed the force threshold. It is important to emphasize that once the user takes over the control, the spring constant between HIP and NIP is increased gradually, and the force applied to the user through the haptic device builds up smoothly and slowly. This causes the ball (virtually coupled to HIP, see Fig. 1) to approach HIP, reducing the large displacement. Due to our blending approach and the simultaneous reduction in the distance between HIP and the ball, the increase in force magnitude was easily handled by the users. During our experiments, the subjects have not reported any instabilities or oscillations in the force response of the device.

In [25], we observed that the role exchange mechanism presents the users with an option to choose and optimize between accuracy and energy when the users were not given any information on how to use the underlying mechanism. In the current study, we explained the role exchange mechanism to the users first and then evaluated their performance. Our results suggest that the proposed role exchange mechanism (RE) improves task performance when compared to the equal control guidance scheme (EC). Also, we observed that the efficiency of the users and the joint efficiency of the dyad are significantly higher under $\mathrm{RE}^{3}$. This implies that the users accomplish a higher amount of work with less effort when they are capable of exchanging roles with the computer. In contrast to [25], this result shows that the users can effectively benefit from a role exchange mechanism when they are explicitly instructed on the principles of interacting with the computer.

Additionally, we sought the benefits of supplementing the system with additional visual and vibrotactile cues to inform the users on the control state regarding the negotiation process. With the integration of these cues (VHC), we observed that task performance deteriorates, probably due to an extra cognitive load intro-

3. Note that the collected data contained outliers, which are addressed before analyzing the data. The trends in our results remain intact regardless of whether we apply outlier elimination or not. In case no outliers are eliminated, we fail to observe any statistically significant differences between the performances achived under different experimental conditions. On the other hand, the conclusions about the efficiency do not change. duced by these cues. However, subjectively, the users reported that these additional cues made the interface of the system easier to use, the task more interactive, and their computer partner more trusted. Under both $\mathrm{RE}$ and VHC, we observed that the movement of the ball was predominantly controlled by the computer. Moreover, the role exchanges were performed at similar instants during the task and their numbers were close under both conditions. However, without the additional cues (under RE), we observed that the users mistakenly thought that they held control of the ball more often then they did under VHC. This is a sign that additional cues were helpful in conveying the control state to the users.

Even though the study presented in this paper focuses only on human-computer cooperation in a virtual task, the proposed mechanism can also enhance the assistive capability of a robotic partner in physical cooperation with humans. In physical cooperation, two humans communicate dominantly through forces for negotiating action plans for accomplishing a task. However, in the context of human-robot interaction, communication through the haptic channel has not been explored in sufficient detail yet. We suggest that as the robots are being more capable of performing a broader variety of tasks, more sophisticated robotic partners that can recognize and respond to the force signals acquired from the humans, will be built. Recently, we have investigated how a dynamic role exchange mechanism adds to the physical cooperation between a human and a robot. The details of this study can be found in [29].

As future work, we would like to investigate the utility of statistical learning models to explore the role of human characteristics in collaboration. We hypothesize that certain human characteristics, such as aggressiveness, submissiveness, and the levels in between, exist in human-computer collaboration. Discovering such characteristics can be beneficial to alter the extent of guidance provided by the collaborating partner (human or computer) and also to program the computer partner to display more human-like behavior since the results of the questionnaire show that no guidance mechanism as is was able to generate this effect.

\section{ACKNOWLEDGMENTS}

We would like to thank Prof. Dr. Fatin Sezgin for his guidance and suggestions throughout the statistical analyses of this study.

\section{REFERENCES}

[1] P. Dourish and V. Bellotti, "Awareness and coordination in shared workspaces," in Proceedings of the 1992 ACM conference on Computer-supported cooperative work, ser. CSCW '92. New York, NY, USA: ACM, 1992, pp. 107-114. 
[2] E. Subasi and C. Basdogan, "A new haptic interaction and visualization approach for rigid molecular docking in virtual environments," Presence: Teleoper. Virtual Environ., vol. 17, no. 1, pp. 73-90, 2008

[3] M. R. McGee, P. D. Gray, and S. A. Brewster, "The effective combination of haptic and auditory textural information," in Proceedings of the First International Workshop on Haptic HumanComputer Interaction, 2001, pp. 118-126.

[4] I. Díaz, J. Hernantes, I. Mansa, A. Lozano-Rodero, D. Borro, J. J. Gil, and E. Sánchez, "Influence of multisensory feedback on haptic accessibility tasks," Virtual Reality, vol. 10, no. 1, pp. 31-40, 2006.

[5] A. Kucukyilmaz, T. Sezgin, and C. Basdogan, "Conveying intentions through haptics in human-computer collaboration," in WHC'11: IEEE World Haptics Conference. IEEE Computer Society, June 2011, pp. 421-426.

[6] R. Groten, D. Feth, A. Peer, and M. Buss, "Shared decision making in a collaborative task with reciprocal haptic feedback - an efficiency-analysis," in IEEE International Conference on Robotics and Automation (ICRA), 2010, pp. 1834-1839.

[7] D. Morris, H. Tan, F. Barbagli, T. Chang, and K. Salisbury, "Haptic feedback enhances force skill learning," in WHC'07: World Haptics Conference. Washington, DC, USA: IEEE Computer Society, 2007, pp. 21-26.

[8] D. Feygin, M. Keehner, and F. Tendick, "Haptic guidance: Experimental evaluation of a haptic training method for a perceptual motor skill," in HAPTICS '02: Proceedings of the 10th Symposium on Haptic Interfaces for Virtual Environment and Teleoperator Systems. Washington, DC, USA: IEEE Computer Society, 2002, pp. 40-47.

[9] I. Oakley, S. Brewster, and P. Gray, "Can you feel the force? an investigation of haptic collaboration in shared editors," in In Proceedings of Eurohaptics, 2001, pp. 54-59.

[10] J. Moll and E.-L. Sallnäs, "Communicative functions of haptic feedback," in Proceedings of the 4th International Conference on Haptic and Audio Interaction Design, ser. HAID '09. Berlin, Heidelberg: Springer-Verlag, 2009, pp. 1-10.

[11] B. A. C. Forsyth and K. E. MacLean, "Predictive haptic guidance: Intelligent user assistance for the control of dynamic tasks," IEEE Transactions on Visualization and Computer Graphics, vol. 12, no. 1, pp. 103-113, 2006.

[12] J. Huegel and M. O'Malley, "Progressive haptic and visual guidance for training in a virtual dynamic task," in Haptics Symposium, 2010 IEEE, mar. 2010, pp. 343-350.

[13] J. Lee and S. Choi, "Effects of haptic guidance and disturbance on motor learning: Potential advantage of haptic disturbance," in Haptics Symposium, 2010 IEEE, march 2010, pp. 335-342.

[14] M. K. O'Malley, A. Gupta, M. Gen, and Y. Li, "Shared control in haptic systems for performance enhancement and training," Journal of Dynamic Systems, Measurement, and Control, vol. 128, no. 1, pp. 75-85, 2006.

[15] J. Crandall and M. Goodrich, "Experiments in adjustable autonomy," in Systems, Man, and Cybernetics, 2001 IEEE International Conference on, vol. 3, 2001, pp. 1624-1629.

[16] M. Sierhuis, J. M. Bradshaw, A. Acquisti, R. van Hoof, R. Jeffers, and A. Uszok, "Human-agent teamwork and adjustable autonomy in practice," in Proceedings of the Seventh International Symposium on Artificial Intelligence, Robotics and Automation in Space (I-SAIRAS), 2003.

[17] S. Nudehi, R. Mukherjee, and M. Ghodoussi, "A sharedcontrol approach to haptic interface design for minimally invasive telesurgical training," Control Systems Technology, IEEE Transactions on, vol. 13, no. 4, pp. 588-592, jul. 2005.

[18] K. B. Reed and M. A. Peshkin, "Physical collaboration of human-human and human-robot teams," IEEE Trans. Haptics, vol. 1, no. 2, pp. 108-120, 2008.

[19] D. Feth, R. Groten, A. Peer, S. Hirche, and M. Buss, "Performance related energy exchange in haptic human-human interaction in a shared virtual object manipulation task," in WHC'09: World Haptics Conference. Washington, DC, USA: IEEE Computer Society, 2009, pp. 338-343.

[20] N. Stefanov, A. Peer, and M. Buss, "Role determination in human-human interaction," in WHC'09: World Haptics Conference. Washington, DC, USA: IEEE Computer Society, 2009, pp. 51-56.

[21] P. Evrard and A. Kheddar, "Homotopy switching model for dyad haptic interaction in physical collaborative tasks," in
WHC'09: World Haptics Conference. Washington, DC, USA: IEEE Computer Society, 2009, pp. 45-50.

[22] B. Corteville, E. Aertbeliën, H. Bruyninckx, J. D. Schutter, and H. V. Brussel, "Human-inspired robot assistant for fast point-to-point movements," in IEEE International Conference on Robotics and Automation, ICRA, 2007, pp. 3639-3644.

[23] V. Duchaine and C. M. Gosselin, "General model of humanrobot cooperation using a novel velocity based variable impedance control," in WHC'07: World Haptics Conference, march 2007, pp. 446-451.

[24] J. Kelso, Human Motor Behavior: An Introduction. Lawrence Erlbaum Associates, 1982.

[25] S. Oguz, A. Kucukyilmaz, T. Sezgin, and C. Basdogan, "Haptic negotiation and role exchange for collaboration in virtual environments," in IEEE Haptics Symposium, mar. 2010, pp. 371378.

[26] C. D. Wickens, J. D. Lee, Y. Liu, and S. Gordon-Becker, An Introduction to Human Factors Engineering, 2nd ed. Prentice Hall, November 1997, vol. 1, no. 1.

[27] R. Groten, D. Feth, R. Klatzky, A. Peer, and M. Buss, “Efficiency analysis in a collaborative task with reciprocal haptic feedback," in IROS'09: Proceedings of the 2009 IEEE/RSI international conference on Intelligent robots and systems. Piscataway, NJ, USA: IEEE Press, 2009, pp. 461-466.

[28] C. Basdogan, C.-H. Ho, M. A. Srinivasan, and M. Slater, “An experimental study on the role of touch in shared virtual environments," ACM Trans. Comput.-Hum. Interact., vol. 7, no. 4 , pp. $443-460,2000$.

[29] A. Moertl, M. Lawitzky, A. Kucukyilmaz, T. Sezgin, C. Basdogan, and S. Hirche, "The role of roles: Physical cooperation between humans and robots," International Journal of Robotics Research (accepted), 2012.

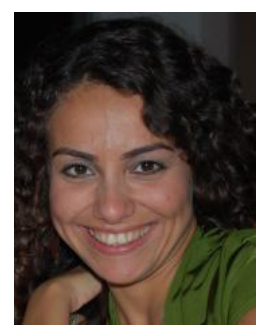

Ayse Kucukyilmaz received her BS and MS degrees in Computer Engineering from Bilkent University, Turkey. In 2008, she started to pursue a $\mathrm{PhD}$ in Computational Sciences and Engineering at Koc University. She has been a member of the Robotics and Mechatronics Laboratory and the Intelligent User Interfaces Laboratory since then. Her current research interests include natural human-computer interaction in virtual domains, haptic user interfaces, affective computing, and machine learning applications in adaptive and immersive multimodal user interfaces.

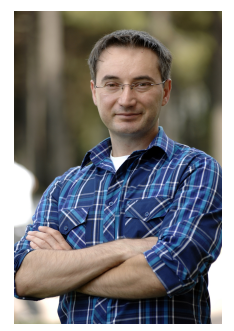

Tevfik Metin Sezgin graduated summacum-laude with Honors from Syracuse University in 1999. He received his MS and $\mathrm{PhD}$ degrees from Massachusetts Institute of Technology in 2001 and 2006 . He subsequently joined the University of Cambridge as a Postdoctoral Research Associate, and held a visiting researcher position at Harvard University in 2010 . He is currently an assistant professor at Koc University, Istanbul. His research interests include intelligent humancomputer interfaces and $\mathrm{HCl}$ applications of machine learning.

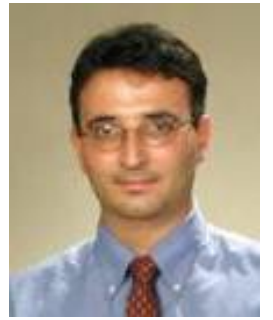

Cagatay Basdogan is a faculty member in the Mechanical Engineering and Computational Sciences and Engineering programs of Koc University, Istanbul, Turkey. He is also the director of the Robotics and Mechatronics Laboratory at Koc University. Before joining Koc University, he worked at NASAJPL/Caltech, MIT, and Northwestern University Research Park. His research interests include haptic interfaces, robotics, mechatronics, biomechanics, medical simulation, computer graphics, and multi-modal virtual environments. Basdogan has a PhD in Mechanical Engineering from Southern Methodist University in 1994. He is currently the associate editor of IEEE Transactions on Haptics and Computer Animation and Virtual Worlds journals. 